\title{
Weighted Hardy operators in local generalized Orlicz-Morrey spaces
}

\author{
Aykol C. ${ }^{1, 凶}$, Azizova Z.O. ${ }^{2}$, Hasanov J.J. ${ }^{2}$ \\ In this paper, we find sufficient conditions on general Young functions $(\Phi, \Psi)$ and the func- \\ tions $\left(\varphi_{1}, \varphi_{2}\right)$ ensuring that the weighted Hardy operators $A_{\omega}^{\alpha}$ and $\mathcal{A}_{\omega}^{\alpha}$ are of strong type from a lo- \\ cal generalized Orlicz-Morrey space $M_{\Phi, \varphi_{1}}^{0, l o c}\left(\mathbb{R}^{n}\right)$ into another local generalized Orlicz-Morrey space \\ $M_{\Psi, \varphi_{2}}^{0, l o c}\left(\mathbb{R}^{n}\right)$. We also obtain the boundedness of the commutators of $A_{\omega}^{\alpha}$ and $\mathcal{A}_{\omega}^{\alpha}$ from $M_{\Phi, \varphi_{1}}^{0, \text { loc }}\left(\mathbb{R}^{n}\right)$ \\ to $M_{\Psi, \varphi_{2}}^{0, l o c}\left(\mathbb{R}^{n}\right)$. \\ Key words and phrases: weighted Hardy operator, local generalized Orlicz-Morrey space, local \\ $B M O$ space.
}

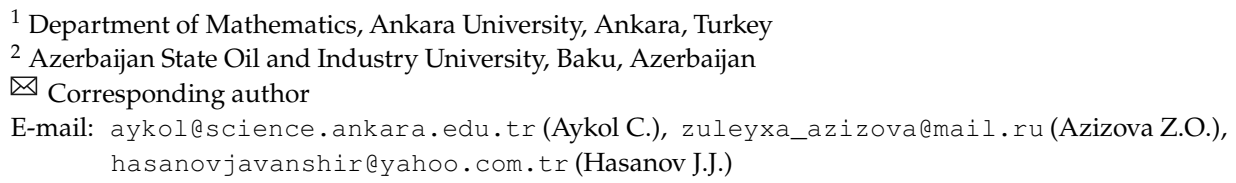

\section{Introduction}

The well known Morrey spaces $L_{p, \lambda}$, introduced in [15], were widely investigated during last decades, including the study of classical operators of harmonic analysis - maximal, singular and potential operators. Hardy operators, maximal functions and singular integrals play a key role in harmonic analysis since maximal functions could control crucial quantitative information concerning the given functions, despite their larger size, while singular integrals, Hilbert transform as it's prototype, nowadays intimately connected with PDE, operator theory and other fields. C.B. Morrey found that many properties of solutions to PDE can be attributed to the boundedness of such operators on Morrey spaces. The generalized Morrey spaces $L^{p, \varphi}$ are obtained by replacing $r^{\lambda}$ by a function $\varphi(r)$ in the definition of the Morrey space. During the last decades vaious classical operators, such as maximal, singular and potential operators and their commutators with $B M O$ functions were widely investigated in both classical and generalized Morrey spaces. For the boundedness of the Hardy-Littlewood maximal operator and fractional integral operators, see for example $[1,4,5,14,16,21,22,24,25]$.

They are useful tools to study harmonic analysis and its applications. For example, the Hardy-Littlewood maximal operator is bounded on $L_{p}$ for $1<p \leq \infty$, but not bounded on $L_{1}$. Using Orlicz spaces, we can investigate the boundedness of the operator near $p=1$ precisely. Characterization of Young functions $A$ for which the Hardy-Littlewood maximal operator or the Hilbert and Riesz transforms are of weak or strong type in Orlicz space $L_{A}$ is known (see for example [6,12]). For the theory of Orlicz spaces, see [6,9-11,20,23]. 
In [17], the author introduced Orlicz-Morrey spaces to investigate the boundedness of generalized fractional integral operators. Orlicz-Morrey spaces unify Orlicz and Morrey spaces. Recently, using Orlicz-Morrey spaces, Y. Sawano, S. Sugano and H. Tanaka [26] proved a Trudinger type inequality for Morrey spaces.

Inequalities involving classical operators of harmonic analysis, such as maximal functions, fractional integrals and singular integrals of convolution type have been extensively investigated in various function spaces. Results on weak and strong type inequalities for operators of this kind in Lebesgue spaces are classical and can be found for example in $[3,27,28]$. Generalizations of these results to Zygmund spaces are presented in [3]. Orlicz-Morrey spaces and maximal and singular operators in such spaces were studied in $[7,8,18,19]$. The boundedness of weighted Hardy operator and its commutators in Orlicz-Morrey spaces was proved in [2].

In this paper we consider the following weighted Hardy operators

$$
A_{\omega}^{\alpha} f(x)=|x|^{\alpha-n} \omega(|x|) \int_{|y| \leq|x|} \frac{f(y)}{\omega(|y|)} d y \quad \text { and } \quad \mathcal{A}_{\omega}^{\alpha} f(x)=|x|^{\alpha} \omega(|x|) \int_{|y|>|x|} \frac{f(y) d y}{|y|^{n} \omega(|y|)}
$$

where $\alpha \geq 0$ and $\omega$ is a weight.

Note that Hardy operators in Orlicz-Morrey spaces were less studied in comparison with maximal, singular and potential operators.

As is well known, the boundedness of commutators of various operators, such as maximal and singular operators, is of importance in applications to PDE. Such boundedness was not studied in local generalized Orlicz-Morrey spaces. We obtain conditions, for the weighted boundedness of the commutators of Hardy operators with BMO functions in local generalized Orlicz-Morrey spaces.

The main purpose of this paper is to find sufficient conditions on general Young functions $(\Phi, \Psi)$ and the functions $\left(\varphi_{1}, \varphi_{2}\right)$ ensuring that the weighted Hardy operators $A_{\omega}^{\alpha}$ and $\mathcal{A}_{\omega}^{\alpha}$ are of strong type from a local generalized Orlicz-Morrey space $M_{\Phi, \varphi_{1}}^{0, l o c}\left(\mathbb{R}^{n}\right)$ into another local generalized Orlicz-Morrey space $M_{\Psi, \varphi_{2}}^{0, l o c}\left(\mathbb{R}^{n}\right)$. We also obtain the boundedness of the commutators of $A_{\omega}^{\alpha}$ and $\mathcal{A}_{\omega}^{\alpha}$ from $M_{\Phi, \varphi_{1}}^{0, l o c}\left(\mathbb{R}^{n}\right)$ to $M_{\Psi, \varphi_{2}}^{0, l o c}\left(\mathbb{R}^{n}\right)$. The main results are given in Theorems $2,3,4$, 5 and 6 .

In the next section, we recall the definitions of Morrey and Orlicz spaces and give the definition of local Orlicz-Morrey and local generalized Orlicz-Morrey spaces. In Section 3, we prove the boundedness of weighted Hardy operators $A_{\omega}^{\alpha}$ and $\mathcal{A}_{\omega}^{\alpha}$ from $M_{\Phi, \varphi_{1}}^{0, \text { loc }}\left(\mathbb{R}^{n}\right)$ to $M_{\Psi, \varphi_{2}}^{0, l o c}\left(\mathbb{R}^{n}\right)$, and in Section 4 , we prove the boundedness of the commutators of $A_{\omega}^{\alpha}$ and $\mathcal{A}_{\omega}^{\alpha}$ from $M_{\Phi, \varphi_{1}}^{0, l o c}\left(\mathbb{R}^{n}\right)$ to $M_{\Psi, \varphi_{2}}^{0, \text { loc }}\left(\mathbb{R}^{n}\right)$.

Throughout the paper we use the letter $C$ for a positive constant, independent of appropriate parameters and not necessary the same at each occurrence. By $A \lesssim B$ we mean that $A \leq C B$ with some positive constant $C$. If $A \lesssim B$ and $B \lesssim A$, we write $A \approx B$ and say that $A$ and $B$ are equivalent.

\section{Preliminaries}

Morrey spaces were introduced by Morrey [15] in 1938 in connection with certain problems in elliptic partial differential equations and calculus of variations. Later, Morrey spaces found important applications to Navier-Stokes and Schrödinger equations, elliptic problems with discontinuous coefficients, and potential theory. 
Definition 1. We denote by $L_{p, \lambda}\left(\mathbb{R}^{n}\right)$ Morrey space for $0 \leq \lambda \leq n, 1 \leq p<\infty, f \in L_{p, \lambda}\left(\mathbb{R}^{n}\right)$ if $f \in L_{p}^{\text {loc }}\left(\mathbb{R}^{n}\right)$ and

$$
L_{p, \lambda}\left(\mathbb{R}^{n}\right)=\left\{f \in L_{p}^{\text {loc }}\left(\mathbb{R}^{n}\right):\|f\|_{L_{p, \lambda}}:=\sup _{x \in \mathbb{R}^{n}, r>0} r^{-\frac{\lambda}{p}}\|f\|_{L_{p}(B(x, r))}<\infty\right\} .
$$

Here and everywhere in the sequel $B(x, r)$ stands for the ball in $\mathbb{R}^{n}$ of radius $r$ centered at $x$. Let $|B(x, r)|$ be the Lebesgue measure of the ball $B(x, r)$ and $|B(x, r)|=v_{n} r^{n}$, where $v_{n}=|B(0,1)|$. $L_{p, \lambda}\left(\mathbb{R}^{n}\right)$ is an expansion of $L_{p}\left(\mathbb{R}^{n}\right)$ in the sense that $L_{p, 0}\left(\mathbb{R}^{n}\right)=L_{p}\left(\mathbb{R}^{n}\right)$. We also denote by $W L_{p, \lambda} \equiv W L_{p, \lambda}\left(\mathbb{R}^{n}\right)$ the weak Morrey space of all functions $f \in W L_{p}^{\text {loc }}\left(\mathbb{R}^{n}\right)$ for which

$$
\|f\|_{W L_{p, \lambda}}=\sup _{x \in \mathbb{R}^{n}, r>0} r^{-\frac{\lambda}{p}}\|f\|_{W L_{p}(B(x, r))}<\infty,
$$

where $W L_{p}$ denotes the weak $L_{p}$-space.

We recall the definition of Young functions.

Definition 2. A function $\Phi:[0,+\infty] \rightarrow[0,+\infty]$ is called a Young function if $\Phi$ is convex, left-continuous, $\lim _{r \rightarrow+0} \Phi(r)=\Phi(0)=0$ and $\lim _{r \rightarrow+\infty} \Phi(r)=\Phi(+\infty)=+\infty$.

From the convexity and $\Phi(0)=0$ it follows that any Young function is increasing. If there exists $s \in(0,+\infty)$ such that $\Phi(s)=+\infty$, then $\Phi(r)=+\infty$ for $r \geq s$.

We say that $\Phi \in \Delta_{2}$, if for any $a>1$, there exists a constant $C_{a}>0$ such that $\Phi(a t) \leq C_{a} \Phi(t)$ for all $t>0$.

Recall that a function $\Phi$ is said to be quasiconvex if there exist a convex function $\omega$ and a constant $c>0$ such that

$$
\omega(t) \leq \Phi(t) \leq c \omega(c t), \quad t \in[0,+\infty) .
$$

Let $\mathcal{Y}$ be the set of all Young functions $\Phi$ such that

$$
0<\Phi(r)<+\infty \text { for } 0<r<+\infty .
$$

If $\Phi \in \mathcal{Y}$, then $\Phi$ is absolutely continuous on every closed interval in $[0,+\infty)$ and bijective from $[0,+\infty)$ to itself.

Definition 3 (Orlicz space). For a Young function $\Phi$, the set

$$
L_{\Phi}\left(\mathbb{R}^{n}\right)=\left\{f \in L_{1}^{\text {loc }}\left(\mathbb{R}^{n}\right): \int_{\mathbb{R}^{n}} \Phi(k|f(x)|) d x<+\infty \text { for some } k>0\right\}
$$

is called Orlicz space. The space $L_{\Phi}^{\operatorname{loc}}\left(\mathbb{R}^{n}\right)$ endowed with the natural topology is defined as the set of all functions $f$ such that $f \chi_{B} \in L_{\Phi}\left(\mathbb{R}^{n}\right)$ for all balls $B \subset \mathbb{R}^{n}\left(\left\|f \chi_{B}\right\|_{L_{\Phi}\left(\mathbb{R}^{n}\right)}=\|f\|_{L_{\Phi}(B)}\right)$.

Note that $L_{\Phi}\left(\mathbb{R}^{n}\right)$ is a Banach space with respect to the norm

$$
\|f\|_{L_{\Phi}}=\inf \left\{\lambda>0: \int_{\mathbb{R}^{n}} \Phi\left(\frac{|f(x)|}{\lambda}\right) d x \leq 1\right\},
$$

see, for example, [23, Section 3, Theorem 10], so that

$$
\int_{\mathbb{R}^{n}} \Phi\left(\frac{|f(x)|}{\|f\|_{L_{\Phi}}}\right) d x \leq 1
$$


Definition 4. The weak Orlicz space

$$
W L_{\Phi}\left(\mathbb{R}^{n}\right)=\left\{f \in L_{1 \mathrm{oc}}^{1}\left(\mathbb{R}^{n}\right):\|f\|_{W L_{\Phi}}<+\infty\right\}
$$

is defined by the norm

$$
\|f\|_{W L_{\Phi}}=\inf \left\{\lambda>0: \sup _{t>0} \Phi(t) m\left(\frac{f}{\lambda}, t\right) \leq 1\right\}
$$

where $m(f, t)=\left|\left\{x \in \mathbb{R}^{n}:|f(x)|>t\right\}\right|$.

For Young functions $\Phi$ and $\Psi$, we write $\Phi \sim \Psi$ if there exists a constant $C \geq 1$ such that $\Phi\left(C^{-1} r\right) \leq \Psi(r) \leq \Phi(C r)$ for all $r \geq 0$. If $\Phi \approx \Psi$, then $L_{\Phi}\left(\mathbb{R}^{n}\right)=L_{\Psi}\left(\mathbb{R}^{n}\right)$ with equivalent norms. For a Young function $\Phi$ and $0 \leq s \leq+\infty$, let $\Phi^{-1}(s)=\inf \{r \geq 0: \Phi(r)>s\}$ (inf $\varnothing=+\infty)$. If $\Phi \in \mathcal{Y}$, then $\Phi^{-1}$ is the usual inverse function of $\Phi$. We note that $\Phi\left(\Phi^{-1}(r)\right) \leq r \leq \Phi^{-1}(\Phi(r))$ for $0 \leq r<+\infty$.

A Young function $\Phi$ is said to satisfy the $\nabla_{2}$-condition, denoted also by $\Phi \in \nabla_{2}$, if

$$
\Phi(r) \leq \frac{1}{2 k} \Phi(k r), \quad r \geq 0,
$$

for some $k>1$.

For a Young function $\Phi$, the complementary function $\widetilde{\Phi}(r)$ is defined by

$$
\widetilde{\Phi}(r)= \begin{cases}\sup \{r s-\Phi(s): s \in[0, \infty)\}, & r \in[0, \infty) \\ +\infty, & r=+\infty\end{cases}
$$

The complementary function $\widetilde{\Phi}$ is also a Young function and $\widetilde{\Phi}=\Phi$. If $\Phi(r)=r$, then $\widetilde{\Phi}(r)=0$ for $0 \leq r \leq 1$ and $\widetilde{\Phi}(r)=+\infty$ for $r>1$. If $1<p<\infty, 1 / p+1 / p^{\prime}=1$ and $\Phi(r)=r^{p} / p$, then $\widetilde{\Phi}(r)=r^{p^{\prime}} / p^{\prime}$. If $\Phi(r)=e^{r}-r-1$, then $\widetilde{\Phi}(r)=(1+r) \log (1+r)-r$.

Remark 1. Note that $\Phi \in \nabla_{2}$ if and only if $\widetilde{\Phi} \in \Delta_{2}$. Also, if $\Phi$ is a Young function, then $\Phi \in \nabla_{2}$ if and only if $\Phi^{\gamma}$ be quasiconvex for some $\gamma \in(0,1)$ (see, for example, [12, p. 15]).

It is known that

$$
r \leq \Phi^{-1}(r) \widetilde{\Phi}^{-1}(r) \leq 2 r \text { for } r \geq 0 .
$$

Note that Young functions satisfy the properties

$$
\left\{\begin{array} { l } 
{ \Phi ( \alpha t ) \leq \alpha \Phi ( t ) , \text { if } 0 \leq \alpha \leq 1 , } \\
{ \Phi ( \alpha t ) \geq \alpha \Phi ( t ) , \text { if } \alpha > 1 , }
\end{array} \text { and } \quad \left\{\begin{array}{l}
\Phi^{-1}(\alpha t) \geq \alpha \Phi^{-1}(t), \text { if } 0 \leq \alpha \leq 1 \\
\Phi^{-1}(\alpha t) \leq \alpha \Phi^{-1}(t), \text { if } \alpha>1 .
\end{array}\right.\right.
$$

The following analog of the Hölder inequality is well known.

Theorem 1 ([29]). For a Young function $\Phi$ and its complementary function $\widetilde{\Phi}$, the inequality $\|f g\|_{L_{1}\left(\mathbb{R}^{n}\right)} \leq 2\|f\|_{L_{\Phi}}\|g\|_{L_{\widetilde{\Phi}}}$ holds.

The following lemma is valid. See, for example $[3,13]$.

Lemma 1. Let $\Phi$ be a Young function and $B$ be a set in $\mathbb{R}^{n}$ with finite Lebesgue measure. Then

$$
\left\|\chi_{B}\right\|_{W L_{\Phi}\left(\mathbb{R}^{n}\right)}=\left\|\chi_{B}\right\|_{L_{\Phi}\left(\mathbb{R}^{n}\right)}=\frac{1}{\Phi^{-1}\left(|B|^{-1}\right)} .
$$


In the next sections, where we prove our main estimates, we use the following lemma, which follows from Theorem 1, Lemma 1 and inequalities (1).

Lemma 2. For a Young function $\Phi$ and $B=B(x, r)$, the following inequality holds

$$
\|f\|_{L_{1}(B)} \leq 2|B| \Phi^{-1}\left(|B|^{-1}\right)\|f\|_{L_{\Phi}(B)} .
$$

Orlicz-Morrey spaces were defined in [17] while investigating the boundedness of generalized fractional integral operators. Here we define the local Orlicz-Morrey spaces.

Definition 5 (local Orlicz-Morrey space). For a Young function $\Phi$ and $0 \leq \lambda \leq n$, we denote by $L_{\Phi, \lambda}^{0, l o c}\left(\mathbb{R}^{n}\right)$ the local Orlicz-Morrey space, defined as the space of all functions $L_{\Phi}^{\text {loc }}\left(\mathbb{R}^{n}\right)$ with finite quasinorm

$$
\|f\|_{L_{\Phi, \lambda}^{0, l o c}\left(\mathbb{R}^{n}\right)}=\sup _{r>0} \Phi^{-1}\left(r^{-\lambda}\right)\|f\|_{L_{\Phi}(B(0, r))} .
$$

Note that if $\Phi(r)=r^{p}, 1 \leq p<\infty$, then $L_{\Phi, \lambda}^{0, \text { loc }}\left(\mathbb{R}^{n}\right)=L_{p, \lambda}^{0, \text { loc }}\left(\mathbb{R}^{n}\right)$. If $\lambda=0$, then $L_{\Phi, \lambda}^{0, \text { loc }}\left(\mathbb{R}^{n}\right)=$ $L_{\Phi}\left(\mathbb{R}^{n}\right)$.

Definition 6 (generalized Orlicz-Morrey space). For a Young function $\Phi$ and $0 \leq \lambda \leq n$, we denote by $M_{\Phi, \varphi}\left(\mathbb{R}^{n}\right)$ the local generalized Orlicz-Morrey space, defined as the space of all functions $L_{\Phi}^{\text {loc }}\left(\mathbb{R}^{n}\right)$ with finite quasinorm

$$
\|f\|_{M_{\Phi, \varphi}}=\sup _{r>0, x \in \mathbb{R}^{n}} \varphi(x, r)^{-1} \Phi^{-1}\left(r^{-n}\right)\|f\|_{L_{\Phi}(B(x, r))} .
$$

Now we define local generalized Orlicz-Morrey spaces.

Definition 7 (local generalized Orlicz-Morrey space). For a Young function $\Phi$ and $0 \leq \lambda \leq n$, we denote by $M_{\Phi, \varphi}^{0, l o c}\left(\mathbb{R}^{n}\right)$ the local generalized Orlicz-Morrey space, defined as the space of all functions $L_{\Phi}^{\text {loc }}\left(\mathbb{R}^{n}\right)$ with finite quasinorm

$$
\|f\|_{M_{\Phi, \varphi}^{0, l o c}}=\sup _{r>0} \varphi(0, r)^{-1} \Phi^{-1}\left(r^{-n}\right)\|f\|_{L_{\Phi}(B(0, r))} .
$$

Note that $M_{\Phi, \varphi}\left(\mathbb{R}^{n}\right) \subset M_{\Phi, \varphi}^{0, l o c}\left(\mathbb{R}^{n}\right)$ or $\|f\|_{M_{\Phi, \varphi}^{0, l o c}} \leq\|f\|_{M_{\Phi, \varphi}}$.

Definition 8. The space of functions with bounded mean oscillation, $B M O\left(\mathbb{R}^{n}\right)$, consists of those functions $f$ with finite norm

$$
\|f\|_{*}=\sup _{t>0, x \in \mathbb{R}^{n}}|B(x, t)|^{-1} \int_{B(x, t)}\left|f(x)-f_{B(x, t)}\right| d y,
$$

where $f_{B(x, t)}=|B(x, t)|^{-1} \int_{B(x, t)} f(y) d y$.

For Hardy operators, instead of $B M O$, we will use the class of functions introduced in the next definition.

Definition 9. For a Young function $\Phi$, we denote by $B M O_{0, \Phi}\left(\mathbb{R}^{n}\right)$ the local BMO space, defined as the space of all functions $L_{\Phi}^{\text {loc }}\left(\mathbb{R}^{n}\right)$ with finite quasinorm

$$
\|f\|_{0, \Phi}=\sup _{r>0, \sigma \in S^{n-1}} \Phi^{-1}\left(r^{-n}\right)\|f(\cdot)-f(r \sigma)\|_{L_{\Phi}} .
$$




\section{Boundedness of weighted Hardy operators in the spaces $M_{\Phi, \varphi}^{0, l o c}\left(\mathbb{R}^{n}\right)$}

In this section sufficient conditions on the pairs $(\Phi, \Psi)$ and $\left(\varphi_{1}, \varphi_{2}\right)$ for the boundedness of the weighted Hardy operators $A_{\omega}^{\alpha}$ and $\mathcal{A}_{\omega}^{\alpha}$ from one local generalized Orlicz-Morrey space $M_{\Phi, \varphi_{1}}^{0, l o c}\left(\mathbb{R}^{n}\right)$ to another local generalized Orlicz-Morrey space $M_{\Psi, \varphi_{2}}^{0, l o c}\left(\mathbb{R}^{n}\right)$ are obtained. First an auxiliary theorem is proved to obtain main results.

Theorem 2. Let $\Phi$ be Young function and the function $\phi$ satisfy the condition $\phi(r) \leq C \phi(t)$, $\frac{\phi(t)}{\Phi^{-1}\left(t^{-n}\right)} \leq C \frac{\phi(r)}{\Phi^{-1}\left(r^{-n}\right)}, 0<r<t$. Then the inequality

$$
\left\|\phi(|x|) \chi_{B(0, r)}\right\|_{L_{\Phi}} \leq C \int_{0}^{r} \frac{\phi(t)}{\Phi^{-1}\left(t^{-n}\right)} \frac{d t}{t}, \quad r>0,
$$

holds, where $C>0$ does not depend on $r$ and $x$.

Proof. Let $B_{k}=B\left(0,2^{-k} r\right) \backslash B\left(0,2^{-k-1} r\right)$. We get

$$
\left\|\phi(|x|) \chi_{B(0, r)}\right\|_{L_{\Phi}} \leq \sum_{k=0}^{\infty}\left\|\phi(|x|) \chi_{B_{k}}\right\|_{L_{\Phi}} .
$$

Since $\phi(r) \leq C \phi(t), 0<r<t$, then

$$
\sum_{k=0}^{\infty}\left\|\phi(|x|) \chi_{B_{k}}\right\|_{L_{\Phi}} \leq \sum_{k=0}^{\infty} \phi\left(2^{-k} r\right)\left\|\chi_{B\left(0,2^{-k} r\right)}\right\|_{L_{\Phi}} .
$$

By Lemma 1, we obtain

$$
\left\|\phi(|x|) \chi_{B(0, r)}\right\|_{L_{\Phi}} \leq C \sum_{k=0}^{\infty} \frac{\phi\left(2^{-k} r\right)}{\Phi^{-1}\left(\left|B\left(0,2^{-k} r\right)\right|^{-1}\right)} \leq C \int_{0}^{r} \frac{\phi(t)}{\Phi^{-1}\left(t^{-n}\right)} \frac{d t}{t}
$$

and we arrive at (2). The last passage to the integral is verified in the standard way with the use of the monotonicity properties of the function $\frac{\phi(t)}{\Phi^{-1}\left(t^{-n}\right)}$ in $t$, imposed by the assumptions of the lemma on $\phi$ as

$$
\begin{aligned}
\int_{0}^{r} \frac{\phi(t)}{\Phi^{-1}\left(t^{-n}\right)} \frac{d t}{t} & =\sum_{k=0}^{\infty} \int_{2^{-k-1} r}^{2^{-k_{r}}} \frac{\phi(t)}{\Phi^{-1}\left(t^{-n}\right)} \frac{d t}{t} \\
& \geq \sum_{k=0}^{\infty} \frac{\phi\left(2^{-k_{r}}\right)}{\Phi^{-1}\left(\left|B\left(0,2^{-k_{r}}\right)\right|^{-1}\right)} \int_{2^{-k-1_{r}}}^{2^{-k_{r}}} \frac{d t}{t}=\ln 2 \sum_{k=0}^{\infty} \frac{\phi\left(2^{-k_{r}}\right)}{\Phi^{-1}\left(\left|B\left(0,2^{-k_{r}}\right)\right|^{-1}\right)} .
\end{aligned}
$$

Thus the proof of the theorem is completed.

In the following two theorems we give sufficient conditions on general Young functions $(\Phi, \Psi)$ and the functions $\left(\varphi_{1}, \varphi_{2}\right)$ ensuring the boundedness of the weighted Hardy operators $A_{\omega}^{\alpha}$ and $\mathcal{A}_{\omega}^{\alpha}$ from the spaces $M_{\Phi, \varphi_{1}}^{0, l o c}\left(\mathbb{R}^{n}\right)$ to $M_{\Psi, \varphi_{2}}^{0, \text { loc }}\left(\mathbb{R}^{n}\right)$.

Theorem 3. Let $\Phi, \Psi$ be Young functions, $0<\alpha<n, f \in L_{\Phi}^{\operatorname{loc}}\left(\mathbb{R}^{n}\right)$, $\frac{r^{\beta}}{\omega(r)} \leq C \frac{t^{\beta}}{\omega(t)}$, $\varphi_{1}(0, t) / t^{\beta} \omega(t) \leq C \varphi_{1}(0, r) / r^{\beta} \omega(r) 0<r<t, \beta \in \mathbb{R}$ and the functions $\left(\varphi_{1}, \varphi_{2}\right)$ and $(\Phi, \Psi)$ satisfy the conditions

$$
\int_{0}^{r} \frac{t^{n} \varphi_{1}(0, t)}{\omega(t)} \frac{d t}{t} \leq C \frac{r^{n} \varphi_{1}(0, r)}{\omega(r)} \quad \text { and } \quad \int_{0}^{r} \frac{s^{\alpha} \varphi_{1}(0, s)}{\Psi^{-1}\left(s^{-n}\right)} \frac{d s}{s} \leq C \frac{\varphi_{2}(0, r)}{\Psi^{-1}\left(r^{-n}\right)} .
$$

Then the weighted Hardy operator $A_{\omega}^{\alpha}$ is bounded from $M_{\Phi, \varphi_{1}}^{0, l o c}\left(\mathbb{R}^{n}\right)$ to $M_{\Psi, \varphi_{2}}^{0, l o c}\left(\mathbb{R}^{n}\right)$. 
Proof. It is clear that for $x \in B(0, r)$ the inequality

$$
\left|A_{\omega}^{\alpha} f(x)\right| \lesssim|x|^{\alpha-n} \omega(|x|) \int_{|z|<|x|} \frac{|f(z)|}{\omega(|z|)} d z
$$

holds. We have

$$
\int_{|z|<r} \frac{|f(z)|}{\omega(|z|)} d z=\sum_{k=0}^{\infty} \int_{B_{k}} \frac{|f(z)|}{\omega(|z|)} d z,
$$

where $B_{k}=\left\{z: 2^{-k-1} r<|z|<2^{-k_{r}}\right\}$. Making use of the fact that there exists a $\beta$ such that $t^{\beta} \omega(t)$ is almost decreasing, we observe that $1 / \omega(|z|) \leq C / \omega\left(2^{-k} r\right)$ on $B_{k}$. Applying this in (4) and making use of the Hölder inequality, we obtain

$$
\int_{|z|<r} \frac{|f(z)|}{\omega(|z|)} d z \leq \sum_{k=0}^{\infty} \frac{\left(2^{-k_{r}}\right)^{n}}{\omega\left(2^{-k_{r}}\right)} \Phi^{-1}\left(\left(2^{-k_{r}}\right)^{-n}\right)\|f\|_{L_{\Phi}\left(B\left(0,2^{\left.-k_{r}\right)}\right)\right.} .
$$

Therefore

$$
\int_{|z|<r} \frac{|f(z)|}{\omega(|z|)} d z \leq C \sum_{k=1}^{\infty} \frac{\left(2^{-k} r\right)^{n} \varphi_{1}\left(0,2^{-k_{r}}\right)}{\omega\left(2^{-k_{r}}\right)}\|f\|_{M_{\Phi, \varphi_{1}}^{0, l o c} .}
$$

Since the function $\varphi_{1}(0, t) / t^{\beta} \omega(t)$ is almost decreasing with some $\beta$, we obtain

$$
\sum_{k=1}^{\infty} \frac{\left(2^{-k_{r}}\right)^{n} \varphi_{1}\left(0,2^{-k} r\right)}{\omega\left(2^{-k_{r}}\right)} \leq C \sum_{k=0}^{\infty} \int_{2^{-k-1_{r}}}^{2^{-k_{r}}} \frac{t^{n} \varphi_{1}(0, t)}{\omega(t)} \frac{d t}{t}
$$

By (5) and (6), we have

$$
\int_{|z|<r} \frac{|f(z)|}{\omega(|z|)} d z \leq C\|f\|_{M_{\Phi, \varphi_{1}}^{0, l o c}} \int_{0}^{r} \frac{t^{n} \varphi_{1}(0, t)}{\omega(t)} \frac{d t}{t} .
$$

It remains to prove that

$$
\left|A_{\omega}^{\alpha} f(x)\right| \lesssim\|f\|_{M_{\Phi, \varphi_{1}}^{0, l o c}}|x|^{\alpha-n} \omega(|x|) \int_{0}^{|x|} \frac{t^{n} \varphi_{1}(0, t)}{\omega(t)} \frac{d t}{t} .
$$

Hence by (3) and Theorem 2 we obtain

$$
\begin{aligned}
\left\|A_{\omega}^{\alpha} f\right\|_{L_{\Psi}(B(0, r))} & \leq C\|f\|_{M_{\Phi, \varphi_{1}}^{0, l o c}}\left\||x|^{\alpha-n} \omega(|x|) \int_{0}^{|x|} \frac{t^{n} \varphi_{1}(0, t)}{\omega(t)} \frac{d t}{t}\right\|_{L_{\Psi}(B(0, r))} \\
& \leq C\|f\|_{M_{\Phi, \varphi_{1}}^{0, l o c}}\left\||x|^{\alpha-n} \omega(|x|) \frac{|x|^{n} \varphi_{1}(0,|x|)}{\omega(|x|)}\right\|_{L_{\Psi}(B(0, r))} \\
& \leq C\|f\|_{M_{\Phi, \varphi_{1}}^{0, l o c}}\left\||x|^{\alpha} \varphi_{1}(0,|x|)\right\|_{L_{\Psi}(B(0, r))} \\
& \leq C\|f\|_{M_{\Phi, \varphi_{1}}^{0, l o c}} \int_{0}^{r} \frac{s^{\alpha} \varphi_{1}(0, s)}{\Psi^{-1}\left(s^{-n}\right)} \frac{d s}{s} \leq C\|f\|_{M_{\Phi, \varphi_{1}}^{0, l o c}} \frac{\varphi_{2}(0, r)}{\Psi^{-1}\left(r^{-n}\right)} .
\end{aligned}
$$

Therefore we get

$$
\left\|A_{\omega}^{\alpha} f\right\|_{L_{\Psi}(B)} \lesssim\|f\|_{M_{\Phi, \varphi_{1}}^{0, l o c}} \frac{\varphi_{2}(0, r)}{\Psi-1\left(r^{-n}\right)} .
$$

Thus the proof of the theorem is completed. 
Theorem 4. Let $\Phi$, $\Psi$ be Young functions, $0<\alpha<n, \frac{r^{\beta}}{\omega(r)} \leq C \frac{t^{\beta}}{\omega(t)}, r^{\alpha} \varphi_{1}(0, r) \Phi^{-1}\left(r^{-n}\right) \leq$ $C t^{\alpha} \varphi_{1}(0, t) \Phi^{-1}\left(t^{-n}\right), 0<r<t, \beta \in \mathbb{R}$ and the functions $\left(\varphi_{1}, \varphi_{2}\right)$ and $(\Phi, \Psi)$ satisfy the conditions

$$
\int_{r}^{\infty} \frac{\varphi_{1}(0, t) \Phi^{-1}\left(t^{-n}\right)}{\omega(t)} \frac{d t}{t} \leq C \frac{\varphi_{1}(0, r) \Phi^{-1}\left(r^{-n}\right)}{\omega(r)}
$$

and

$$
\int_{0}^{r} \frac{t^{\alpha} \varphi_{1}(0, t) \Phi^{-1}\left(t^{-n}\right)}{\Psi^{-1}\left(t^{-n}\right)} \frac{d t}{t} \leq C \frac{\varphi_{2}(0, r)}{\Psi^{-1}\left(r^{-n}\right)}
$$

where $C$ does not depend on $r$. Then the weighted Hardy operator $\mathcal{A}_{\omega}^{\alpha}$ is bounded from $M_{\Phi, \varphi_{1}}^{0, \text { loc }}\left(\mathbb{R}^{n}\right)$ to $M_{\Psi, \varphi_{2}}^{0, \text { loc }}\left(\mathbb{R}^{n}\right)$.

Proof. It is clear that for $x \in B(0, r)$ the inequality

$$
\left|\mathcal{A}_{\omega}^{\alpha} f(x)\right| \lesssim|x|^{\alpha} \omega(|x|) \int_{|z|>|x|} \frac{|f(z)|}{|z|^{n} \omega(|z|)} d z
$$

holds. By Fubini's theorem we have

$$
\int_{|z|>r} \frac{|f(z)|}{|z|^{n} \omega(|z|)} d z \approx \int_{|z|>r} \frac{|f(z)|}{\omega(|z|)}\left(\int_{|z|}^{\infty} \frac{d t}{t^{n+1}}\right) d z \approx \int_{r}^{\infty}\left(\int_{r<|z|<t} \frac{|f(z)|}{\omega(|z|)} d z\right) \frac{d t}{t^{n+1}} .
$$

By Lemma 2 we get

$$
\begin{aligned}
\int_{|z|>r} \frac{|f(z)|}{\omega(|z|)} d z \lesssim \int_{r}^{\infty} \frac{1}{\omega(t)}\left(\int_{|z|<t}|f(z)| d z\right) \frac{d t}{t^{n+1}} & \lesssim \int_{r}^{\infty} \frac{t^{n}}{\omega(t)} \Phi^{-1}\left(t^{-n}\right)\|f\|_{L_{\Phi}(B(0, t))} \frac{d t}{t^{n+1}} \\
& \lesssim \int_{r}^{\infty} \frac{1}{\omega(t)} \Phi^{-1}\left(t^{-n}\right)\|f\|_{L_{\Phi}(B(0, t))} \frac{d t}{t} .
\end{aligned}
$$

Moreover,

$$
\left|\mathcal{A}_{\omega}^{\alpha} f(x)\right| \leq C|x|^{\alpha} \omega(|x|) \int_{|x|}^{\infty} \frac{1}{\omega(t)} \Phi^{-1}\left(t^{-n}\right)\|f\|_{L_{\Phi}(B(0, t))} \frac{d t}{t} .
$$

By (7), the inequality

$$
\begin{aligned}
& \left|\mathcal{A}_{\omega}^{\alpha} f(x)\right| \leq C|x|^{\alpha} \omega(|x|) \int_{|x|}^{\infty} \frac{1}{\omega(t)} \Phi^{-1}\left(t^{-n}\right)\|f\|_{L_{\Phi}(B(0, t))} \frac{d t}{t} \\
& \leq C\|f\|_{M_{\Phi, \varphi_{1}}^{0,1 o c}}|x|^{\alpha} \omega(|x|) \int_{|x|}^{\infty} \frac{\varphi_{1}(0, t) \Phi^{-1}\left(t^{-n}\right)}{\omega(t)} \frac{d t}{t}
\end{aligned}
$$

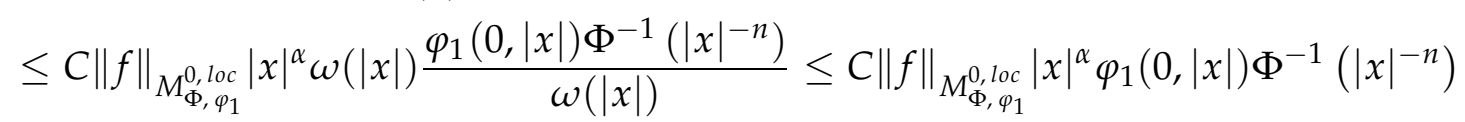

is valid. Therefore by (8) and Theorem 2 we get

$$
\begin{aligned}
\left\|\mathcal{A}_{\omega}^{\alpha} f\right\|_{L_{\Psi}(B(0, r))} & \leq C\|f\|_{M_{\Phi, \varphi_{1}}^{0, l o c}}\left\||x|^{\alpha} \varphi_{1}(0,|x|) \Phi^{-1}\left(|x|^{-n}\right)\right\|_{L_{\Psi}(B(0, r))} \\
& \leq C\|f\|_{M_{\Phi, \varphi_{1}}^{0, l o c}} \int_{0}^{r} \frac{t^{\alpha} \varphi_{1}(0, t) \Phi^{-1}\left(t^{-n}\right)}{\Psi^{-1}\left(t^{-n}\right)} \frac{d t}{t} \leq C\|f\|_{M_{\Phi, \varphi_{1}}^{0, l o c}} \frac{\varphi_{2}(0, r)}{\Psi^{-1}\left(r^{-n}\right)} .
\end{aligned}
$$

Thus the proof of the theorem is completed. 


\section{Boundedness of the commutators of weighted Hardy operators on the spaces $M_{\Phi, \varphi}^{0, l o c}$}

The commutators of weighted Hardy operators are defined by

$$
\left[b, A_{\omega}^{\alpha}\right] f=A_{\omega}^{\alpha}(b f)-b A_{\omega}^{\alpha}(f) .
$$

In the following two theorems we prove the boundedness of commutators of weighted Hardy operators from one local generalized Orlicz-Morrey space $M_{\Phi, \varphi_{1}}^{0, l o c}\left(\mathbb{R}^{n}\right)$ to another local generalized Orlicz-Morrey space $M_{\Psi, \varphi_{2}}^{0, l o c}\left(\mathbb{R}^{n}\right)$.

Theorem 5. Let $\Phi, \Psi$ be Young functions, $0<\alpha<n, f \in L_{\Phi}^{\operatorname{loc}}\left(\mathbb{R}^{n}\right), b \in B M O_{0, \widetilde{\Phi}}\left(\mathbb{R}^{n}\right)$, $\frac{r^{\beta}}{\omega(r)} \leq C \frac{t^{\beta}}{\omega(t)}, \varphi_{1}(0, t) / t^{\beta} \omega(t) \leq C \varphi_{1}(0, r) / r^{\beta} \omega(r) 0<r<t, \beta \in \mathbb{R}$ and the functions $\left(\varphi_{1}, \varphi_{2}\right)$ and $(\Phi, \Psi)$ satisfy the conditions (3).

Then the operator $\left[b, A_{\omega}^{\alpha}\right]$ is bounded from $M_{\Phi, \varphi_{1}}^{0, \text { loc }}\left(\mathbb{R}^{n}\right)$ to $M_{\Psi, \varphi_{2}}^{0, l o c}\left(\mathbb{R}^{n}\right)$.

Proof. It is clear that for $x \in B(0, r)$ we get

$$
\left|\left[b, A_{\omega}^{\alpha}\right] f(x)\right| \lesssim|x|^{\alpha-n} \omega(|x|) \int_{|z|<|x|} \frac{|f(z)||b(x)-b(z)|}{\omega(|z|)} d z .
$$

We have

$$
\int_{|z|<r} \frac{|f(z)|}{\omega(|z|)} d z=\sum_{k=0}^{\infty} \int_{B_{k}} \frac{|f(z)||b(x)-b(z)|}{\omega(|z|)} d z
$$

where $B_{k}=\left\{z: 2^{-k-1} r<|z|<2^{-k} r\right\}$. Making use of the fact that there exists a $\beta$ such that $t^{\beta} \omega(t)$ is almost decreasing, we observe that $1 / \omega(|z|) \leq C / \omega\left(2^{-k_{r}}\right)$ on $B_{k}$. Applying this in (9) and making use of the Hölder inequality, we obtain

$$
\begin{aligned}
\int_{|z|<r} \frac{|f(z)||b(x)-b(z)|}{\omega(|z|)} d z & \leq C \sum_{k=0}^{\infty} \frac{1}{\omega\left(2^{\left.-k_{r}\right)}\right.}\|b(\cdot)-b(x)\|_{L_{\widetilde{\Phi}}\left(B\left(0,2^{\left.-k_{r}\right)}\right)\right.}\|f\|_{L_{\Phi}\left(B\left(0,2^{\left.-k_{r}\right)}\right)\right.} \\
& \leq C\|b\|_{B M O_{0, \tilde{\Phi}}}\|f\|_{M_{\Phi, \varphi_{1}}^{0, l o c}} \sum_{k=0}^{\infty} \frac{\left(2^{-k_{r}}\right)^{n} \varphi_{1}\left(0,2^{\left.-k_{r}\right)}\right.}{\omega\left(2^{\left.-k_{r}\right)}\right.} .
\end{aligned}
$$

Therefore we get

$$
\int_{|z|<r} \frac{|f(z)||b(x)-b(z)|}{\omega(|z|)} d z \leq C\|b\|_{B M O_{0, \tilde{\Phi}}}\|f\|_{M_{\Phi, \varphi_{1}}^{0, l o c}} \sum_{k=1}^{\infty} \frac{\left(2^{-k_{r}}\right)^{n} \varphi_{1}\left(0,2^{-k_{r}}\right)}{\omega\left(2^{-k_{r}}\right)} .
$$

Since the function $\varphi_{1}(0, t) / t^{\beta} \omega(t)$ is almost decreasing with some $\beta$, we obtain

$$
\sum_{k=1}^{\infty} \frac{\left(2^{-k} r\right)^{n} \varphi_{1}\left(0,2^{-k} r\right)}{\omega\left(2^{-k_{r}}\right)} \leq C \sum_{k=0}^{\infty} \int_{2^{-k-1} r}^{2^{-k_{r}}} \frac{t^{n} \varphi_{1}(0, t)}{\omega(t)} \frac{d t}{t} .
$$

By (10) and (11), we have

$$
\int_{|z|<r} \frac{|f(z)||b(x)-b(z)|}{\omega(|z|)} d z \leq C\|b\|_{B M O_{0, \widetilde{\Phi}}}\|f\|_{M_{\Phi, \varphi_{1}}^{0, l o c}} \int_{0}^{r} \frac{t^{n} \varphi_{1}(0, t)}{\omega(t)} \frac{d t}{t} .
$$


It remains to prove that

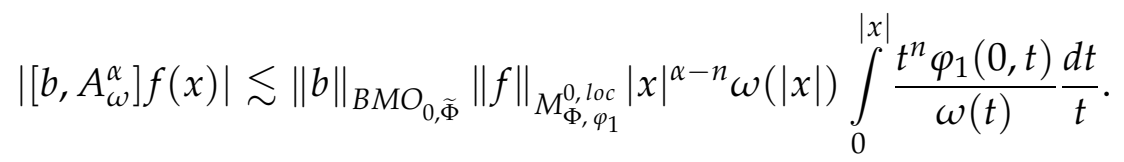

Hence by (3) and Theorem 2, we obtain

$$
\begin{aligned}
\left\|\left[b, A_{\omega}^{\alpha}\right] f\right\|_{L_{\Psi}(B(0, r))} & \leq C\|b\|_{B M O_{0, \tilde{\Phi}}}\|f\|_{M_{\Phi, \varphi_{1}}^{0, l o c}}\left\||x|^{\alpha-n} \omega(|x|) \int_{0}^{|x|} \frac{t^{n} \varphi_{1}(0, t)}{\omega(t)} \frac{d t}{t}\right\|_{L_{\Psi}(B(0, r))} \\
& \leq C\|b\|_{B M O_{0, \tilde{\Phi}}}\|f\|_{M_{\Phi, \varphi_{1}}^{0, l o c}}\left\||x|^{\alpha-n} \omega(|x|) \frac{|x|^{n} \varphi_{1}(0,|x|)}{\omega(|x|)}\right\|_{L \Psi(B(0, r))} \\
& \leq C\|b\|_{B M O_{0, \tilde{\Phi}}}\|f\|_{M_{\Phi, \varphi_{1}}^{0, l o c}}\left\||x|^{\alpha} \varphi_{1}(0,|x|)\right\|_{L_{\Psi}(B(0, r))} \\
& \leq C\|b\|_{B M O_{0, \tilde{\Phi}}}\|f\|_{M_{\Phi, \varphi_{1}}^{0, l o c}} \int_{0}^{r} \frac{s^{\alpha} \varphi_{1}(0, s)}{\Psi-1\left(s^{-n}\right)} \frac{d s}{s} \leq C\|f\|_{M_{\Phi, \varphi_{1}}^{0, l o c}} \frac{\varphi_{2}(0, r)}{\Psi-1\left(r^{-n}\right)}
\end{aligned}
$$

Consequently we obtain

$$
\left\|\left[b, A_{\omega}^{\alpha}\right] f\right\|_{L_{\Psi}(B)} \lesssim\|b\|_{B M O_{0, \widetilde{\Phi}}}\|f\|_{M_{\Phi, \varphi_{1}}^{0, l o c}} \frac{\varphi_{2}(0, r)}{\Psi-1\left(r^{-n}\right)}
$$

Therefore the operator $\left[b, A_{\omega}^{\alpha}\right]$ is bounded from $M_{\Phi, \varphi_{1}}^{0, l o c}\left(\mathbb{R}^{n}\right)$ to $M_{\Psi, \varphi_{2}}^{0, l o c}\left(\mathbb{R}^{n}\right)$.

Theorem 6. Let $\Phi, \Psi$ be Young functions, $0<\alpha<n, \frac{r^{\beta}}{\omega(r)} \leq C \frac{t^{\beta}}{\omega(t)}, 0<r<t, \beta \in \mathbb{R}$, $b \in B M O_{0, \widetilde{\Phi}}\left(\mathbb{R}^{n}\right), r^{\alpha} \varphi_{1}(0, r) \Phi^{-1}\left(r^{-n}\right) \leq C t^{\alpha} \varphi_{1}(0, t) \Phi^{-1}\left(t^{-n}\right), 0<r<t$, the functions $\left(\varphi_{1}, \varphi_{2}\right)$ and $(\Phi, \Psi)$ satisfy the conditions

$$
\int_{r}^{\infty} \frac{\varphi_{1}(0, t)}{\omega(t)} \frac{d t}{t} \leq C \frac{\varphi_{1}(0, r)}{\omega(r)} \quad \text { and } \quad \int_{0}^{r} \frac{t^{\alpha} \varphi_{1}(0, t)}{\Psi^{-1}\left(t^{-n}\right)} \frac{d t}{t} \leq C \frac{\varphi_{2}(0, r)}{\Psi^{-1}\left(r^{-n}\right)}
$$

Then the operator $\left[b, \mathcal{A}_{\omega}^{\alpha}\right]$ is bounded from $M_{\Phi, \varphi_{1}}^{0, l o c}\left(\mathbb{R}^{n}\right)$ to $M_{\Psi, \varphi_{2}}^{0, l o c}\left(\mathbb{R}^{n}\right)$.

Proof. It is clear that since $x \in B(0, r)$, we get

$$
\left|\left[b, \mathcal{A}_{\omega}^{\alpha}\right] f(x)\right| \lesssim|x|^{\alpha} \omega(|x|) \int_{|z|>|x|} \frac{|f(z)||b(x)-b(z)|}{|z|^{n} \omega(|z|)} d z
$$

By Fubini's theorem we have

$$
\begin{aligned}
\int_{|z|>|x|} \frac{|f(z)||b(x)-b(z)|}{|z|^{n} \omega(|z|)} d z & \approx \int_{|z|>|x|} \frac{|f(z)||b(x)-b(z)|}{\omega(|z|)}\left(\int_{|z|}^{\infty} \frac{d t}{t^{n+1}}\right) d z \\
& \approx \int_{|x|}^{\infty}\left(\int_{|x|<|z|<t} \frac{|f(z)||b(x)-b(z)|}{\omega(|z|)} d z\right) \frac{d t}{t^{n+1}}
\end{aligned}
$$


Therefore we obtain

$$
\begin{aligned}
& \left|\left[b, \mathcal{A}_{\omega}^{\alpha}\right] f(x)\right| \lesssim|x|^{\alpha} \omega(|x|) \int_{|x|}^{\infty} \frac{1}{\omega(t)}\left(\int_{|z|<t}|f(z)||b(x)-b(z)| d z\right) \frac{d t}{t^{n+1}} \\
& \lesssim|x|^{\alpha} \omega(|x|) \int_{|x|}^{\infty} \frac{1}{\omega(t)}\|f\|_{L_{\Phi}(B(0, t))}|| b(x)-b(\cdot) \|_{L_{\widetilde{\Phi}}(B(0, t))} \frac{d t}{t^{n+1}}
\end{aligned}
$$

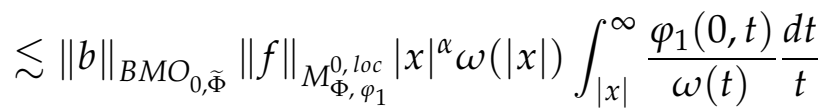

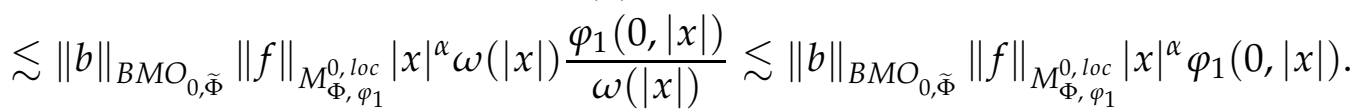

Finally we get

$$
\begin{aligned}
\left\|\left[b, \mathcal{A}_{\omega}^{\alpha}\right] f\right\|_{L \Psi(B(0, r))} & \leq C\|b\|_{B M O_{0, \tilde{\Phi}}}\|f\|_{M_{\Phi, \varphi_{1}}^{0, l o c}}\left\||x|^{\alpha} \varphi_{1}(0,|x|)\right\|_{L \Psi(B(0, r))} \\
& \leq C\|b\|_{B M O_{0, \tilde{\Phi}}}\|f\|_{M_{\Phi, \varphi_{1}}^{0, l o c}} \int_{0}^{r} \frac{t^{\alpha} \varphi_{1}(0, t)}{\Psi^{-1}\left(t^{-n}\right)} \frac{d t}{t} \leq C\|b\|_{B M O_{0, \tilde{\Phi}}}\|f\|_{M_{\Phi, \varphi_{1}}^{0, l o c}} \frac{\varphi_{2}(0, r)}{\Psi^{-1}\left(r^{-n}\right)} .
\end{aligned}
$$

Therefore the operator $\left[b, \mathcal{A}_{\omega}^{\alpha}\right]$ is bounded from $M_{\Phi, \varphi_{1}}^{0, l o c}\left(\mathbb{R}^{n}\right)$ to $M_{\Psi, \varphi_{2}}^{0, l o c}\left(\mathbb{R}^{n}\right)$.

\section{References}

[1] Adams D.R. A note on Riesz potentials. Duke Math. J. 1975, 42 (4), 765-778. doi:10.1215/S0012-7094-75-04265-9

[2] Azizova Z.O., Hasanov J.J. The weighted Hardy operator and it is commutator on Orlicz-Morrey spaces. IJPAM 2018, 118 (2), 385-395.

[3] Bennett C., Sharpley R. Interpolation of operators. Academic Press, Boston, 1988.

[4] Burtseva E., Samko N. On weighted generalized fractional and Hardy-type operators acting between Morrey-type spaces. Frac. Calc. Appl. Anal. 2017, 20 (6), 1545-1566. doi:10.1515/fca-2017-0081

[5] Chiarenza F., Frasca M. Morrey spaces and Hardy-Littlewood maximal function. Rend. Mat. Appl. (7) 1987, 7 (7), 273-279.

[6] Cianchi A. Strong and weak type inequalities for some classical operators in Orlicz spaces. J. Lond. Math. Soc. (2) 2016, 60 (1), 187-202. doi:10.1112/S0024610799007711

[7] Guliyev V.S., Deringoz F., Hasanov J.J. $\Phi$-admissible singular operators and their commutators on vanishing generalized Orlicz-Morrey spaces. J. Inequal. Appl. 2014, 2014 (143), 1-18. doi:10.1186/1029-242X-2014-143

[8] Hasanov J.J. $\Phi$-admissible sublinear singular operators and generalized Orlicz-Morrey spaces. J. Funct. Spaces 2014, 2014, 1-8. doi:10.1155/2014/505237

[9] Kita H. On maximal functions in Orlicz spaces. Proc. Amer. Math. Soc. 1996, 124 (10), 3019-3025.

[10] Kita H. On Hardy-Littlewood maximal functions in Orlicz spaces. Math. Nachr. 1997, 183 (1), 135-155. doi:10.1002/mana.19971830109

[11] Kita H. Inequalities with weights for maximal functions in Orlicz spaces. Acta Math. Hungar. 1996, 72 (4), 291-305. doi:10.1007/BF00114542

[12] Kokilashvili V., Krbec M.M. Weighted inequalities in Lorentz and Orlicz Spaces. World Scientific, Singapore, 1991.

[13] Liu P., Wang M. Weak Orlicz spaces: Some basic properties and their applications to harmonic analysis. Sci. China Math. 2013, 56 (4), 789-802. doi:10.1007/s11425-012-4452-5

[14] Lukkassen D., Persson L.-E., Samko S., Wall P. Weighted Hardy-Type Inequalities in Variable Exponent MorreyType Spaces. J. Funct. Spaces 2013, 2013, 1-11. doi:10.1155/2013/716029 
[15] Morrey C.B. On the solutions of quasi-linear elliptic partial differential equations. Trans. Amer. Math. Soc. 1938, 43, 126-166. doi:10.1090/S0002-9947-1938-1501936-8

[16] Nakai E. Hardy-Littlewood maximal operator, singular integral operutors and the Riesz potentials on generalized Morrey spaces. Math. Nachr. 1994, 166 (1), 95-103. doi:10.1002/mana.19941660108

[17] Nakai E. Generalized fractional integrals on Orlicz-Morrey spaces, Banach and Function Spaces (Kitakyushu, 2003). Yokohama Publishers, Yokohama, 2004, 323-333.

[18] Nakai E. Orlicz-Morrey spaces and the Hardy-Littlewood maximal function. Studia Math. 2008, 188 (3), $193-221$. doi:10.4064/sm188-3-1

[19] Nakai E. Calderón-Zygmund operators on Orlicz-Morrey spaces and modular inequalities. Banach and function spaces II, Yokohama Publ., Yokohama, 2008, 393-410.

[20] O'Neil R. Fractional integration in Orlicz spaces. I. Trans. Amer. Math. Soc. 1965, 115, 300-328. doi:10.2307/1994271

[21] Peetre J. On the theory of $\mathcal{L}_{p, \lambda}$ spaces. J. Funct. Anal. 1969, 4 (1), 71-87. doi:10.1016/0022-1236(69)90022-6

[22] Persson L.-E., Ragusa M., Samko N., Wall P. Commutators of Hardy operators in vanishing Morrey spaces. AIP Conf. Proc. 2021, 1493, 859-866. doi: 10.1063/1.4765588

[23] Rao M.M., Ren Z.D. Theory of Orlicz spaces. M. Dekker, New York, 1991.

[24] Samko N. Weighted Hardy and singular operators in Morrey spaces. J. Math. Anal. Appl. 2009,350 (1), $56-72$. doi:10.1016/j.jmaa.2008.09.021

[25] Sawano Y. A handbook of harmonic analysis. Tokyo, 2011.

[26] Sawano Y., Sugano S., Tanaka H. Orlicz-Morrey spaces and fractional operators. Potential Anal. 2012 , 36 (4), 517-556. doi:10.1007/s11118-011-9239-8

[27] Stein E.M. Harmonic Analysis: Real variable methods, orthogonality and oscillatory integrals. Princeton Univ. Press, Princeton NJ, 1993.

[28] Torchinsky A. Real variable methods in harmonic analysis. Pure and Applied Math. 123, Academic Press, New York, 1986.

[29] Weiss G. A note on Orlicz spaces. Portugal Math. 1956, 15 (1), 35-47.

Received 13.03.2020

Revised 22.06.2021

Айкол С., Азізова 3.О., Хасанов Аж.Аж. Зважені оператори Гарді в локальних узагальнених просторах Орліча-Морея // Карпатські матем. публ. - 2021. — Т.13, №2. - С. 522-533.

У цій статті ми знаходимо достатні умови на загальні функції Юнга $(\Phi, \Psi)$ і функції $\left(\varphi_{1}, \varphi_{2}\right)$, що забезпечують строгий тип зважених операторів Гарді $A_{\omega}^{\alpha}$ та $\mathcal{A}_{\omega}^{\alpha}$ з локального узагальненого простору Орліча-Морея $M_{\Phi, \varphi_{1}}^{0, l o c}\left(\mathbb{R}^{n}\right)$ в інший локальний узагальнений простір Орліча-Морея $M_{\Psi, \varphi_{2}}^{0, l o c}\left(\mathbb{R}^{n}\right)$. Ми також отримуємо обмеженість комутаторів операторів $A_{\omega}^{\alpha}$ та $\mathcal{A}_{\omega}^{\alpha}$ з $M_{\Phi, \varphi_{1}}^{0, l o c}\left(\mathbb{R}^{n}\right)$ в $M_{\Psi, \varphi_{2}}^{0, l o c}\left(\mathbb{R}^{n}\right)$.

Ключові слова і фрази: зважений оператор Гарді, локальний узагальнений простір ОрлічаМорея, локальний ВМО-простір. 